\title{
Osteoporosis Management in Ankylosing Spondylitis
}

\author{
Alicia M. Hinze, $M D^{*}$ \\ Grant H. Louie, MD, MHS
}

\author{
Address \\ *Division of Rheumatology, Johns Hopkins University School of Medicine, 5200 \\ Eastern Avenue, MFL Building, Center Tower, Suite 4100, Baltimore, MD, 21224, \\ USA \\ Email: alicia.hinze@jhmi.edu
}

Published online: 5 October 2016

(C) Springer International Publishing AG 2016

This article is part of the Topical Collection on Comorbidities

Keywords Ankylosing spondylitis • Bone mineral density • Osteoporosis

\section{Opinion statement}

Low bone mineral density (BMD) is increasingly recognized as a common comorbid condition in ankylosing spondylitis (AS). As low BMD increases fracture risk, it is important to identify and treat low BMD in patients with AS who have been shown to be at increased risk for fractures above the population normal. Since low BMD occurs early in disease, we screen during the first year of diagnosis with dual energy $x$-ray absorptiometry (DXA). If patients are found to have osteoporosis by T-score of less than -2.5 or if their Z-score on DXA is more than two standard deviations below the mean, we initiate therapy with bisphosphonates in males and in females who are not planning any future pregnancies. While reduction in fracture risk with bisphosphonate therapy has not been clearly defined in patients with AS, reduction in vertebral and hip fractures has been well established in primary osteoporosis and thus it is our first-line treatment. If there are contraindications to the use of bisphosphonates in the treatment of low BMD, we will consider the use of denosumab. If the patient is not receiving a TNF-alpha inhibitor (TNFi) and has active disease, we also favor early initiation of TNFi due to their positive effects on BMD though the outcome on reduction in vertebral fractures remains unclear. We counsel all patients regarding the importance of adequate intake of vitamin $D$ and calcium per the Institute of Medicine guidelines. Patients should be encouraged to participate in weight-bearing activities with a focus on core strength and gait training.

\section{Introduction}

Ankylosing spondylitis and low bone mineral density Low bone mineral density (BMD) is a common but underappreciated comorbidity in patients with ankylosing spondylitis (AS), with a prevalence of over
$50 \%$ in patients undergoing screening $[1-4,5 \bullet]$. As the onset of AS typically occurs in the third and fourth decades of life, many of these patients are younger than the traditional cohort of older 
patients with low BMD; thus, low BMD is an easily overlooked comorbid condition in the clinical care of patients with AS. Even patients who have had AS for less than 10 years are at an elevated risk for low BMD [5•]. Interestingly, older age has not been found to be a significant risk factor for low BMD in patients with AS [6].

AS is a chronic inflammatory condition that predominately affects the axial skeleton and potentially leads to spinal fusion. The pathophysiology of AS is one of abnormal bone metabolism characterized by pathological new bone formation in the cortical zones of the vertebrae with loss of trabecular bone in the center of the vertebral bodies. Osteoproliferation in the cortical zones and paravertebral ligaments, called syndesmophytes, leads to increased spinal rigidity. The loss of trabecular bone leads to low BMD. A higher burden of syndesmophytes coupled with low BMD has been associated with an increased risk of vertebral fractures $[7,8]$. Peak fracture risk has been shown to occur as early as 2.5 years after AS diagnosis, which underscores the importance of detecting and treating low BMD early in the disease course to reduce risk factors for vertebral fractures [9]. Studies have shown a higher vertebral fracture risk in patients with AS; however, studies are conflicting regarding the risk of non-vertebral fractures in patients with AS versus controls [9-11].

The standardized method for evaluating low BMD is by dual energy $\mathrm{x}$-ray absorptiometry (DXA) which reports the standard deviation from peak bone mass (T-score) and age-matched normal values (Z-score). The World Health Organization classifies low BMD into the two categories of osteopenia and osteoporosis. Specifically, osteopenia is defined as a T-score between -1 and -2.4 and osteoporosis is defined as a T-score of less than or equal to -2.5 on DXA. The reported Z-scores indicate the standard deviation above or below the population normal by age, sex, weight, and ethnicity and should be used to interpret BMD in pre-menopausal women and men less than age 50. Two standard deviations below the mean is considered below the expected range [12]. Standard views on DXA include the anteroposterior (AP) lumbar spine, femoral neck, and total hip. An alternative imaging site also includes the radius, if the hip and/or spine cannot be used. The United States Preventative Services Task Force (USPSTF) recommends initial screening for osteoporosis in women who are 65 years or older without known fractures or secondary causes of osteoporosis. Women aged less than 65 should be screened if their 10-year fracture risk is equal to or greater than a 65-year-old Caucasian woman without additional risk factors [13]. Clinical risk factors for fracture include advancing age, previous fracture, glucocorticoid therapy, parental history of hip fracture, low body weight, current cigarette smoking, excessive alcohol consumption, rheumatoid arthritis, or secondary causes of osteoporosis (e.g., malabsorption secondary to inflammatory bowel disease, hypogonadism, premature menopause, etc.) [14]. While the USPSTF does not provide recommendations on osteoporosis screening in men, other groups such as the National Osteoporosis Foundation and the Endocrine Society recommend that testing is considered for all men older than 70 years of age, and in men and women 50 to 70 years of age when risk factors are present $[15,16]$.

No specific guidelines exist regarding the optimal time to start screening for low BMD in patients with AS; however, the prevalence of low BMD has been reported to be as high as $54 \%$ in the spine and $51 \%$ in the femoral neck in patients who have had AS for less than 10 years [17]. As a majority of patients are diagnosed with AS between the ages of 20 and 40, they are often well below the recommended age for screening based on current guidelines despite their substantially elevated risk; thus, a high index of suspicion is required. Notably, patients included in the meta-analysis documenting early risk of low BMD had a mean age of 34.5 years and were $87 \%$ male [17]. Creactive protein, perhaps reflecting increased disease activity, has been found to be significantly associated with the presence of low BMD [6]. Based on the high prevalence of low BMD in early AS coupled with the increased vertebral fracture risk seen in early disease, we recommend screening for low BMD in the first year of diagnosis of AS with continued monitoring every 2 years, especially in the setting of high disease activity.

\section{Detection of low BMD in AS}

The standard approach for measuring BMD in the general population is by DXA. However, the presence of syndesmophytes at the lumbar spine may falsely elevate BMD measurements in AS. To 
quantify this potential confounder, several cited studies in this review used the modified Stoke AS Spine Score (mSASSS), which is a radiographic scoring method of damage (i.e., syndesmophytes) along the cervical and lumbar spine. Some studies which have shown an increase in BMD among patients with AS over time have also shown the increase in BMD to correlate with increasing mSASSS, implicating an increase in calcifications from the underlying disease process rather than an increase in trabecular bone density [18 1 . However, these associations have not been seen in all studies $[19,20]$.

An alternative screening method for the detection of osteoporosis in AS includes the lateral lumbar DXA. Lateral lumbar DXA evaluates the trabecular vertebral bone, which is the metabolically active bone. It excludes the more calcified cortical bone, as well as calcified ligaments and degenerative osteophytes that traditional AP views obtain. One Swedish study showed that patients with AS had a significantly lower BMD than age-matched controls on lateral lumbar DXA [21]. Decreasing lateral lumbar BMD correlated significantly with increasing mSASSS. This was in contrast to the AP lumbar BMD, which tended to increase with increasing mSASSS. As population normals for BMD have not been established for the lateral lumbar DXA projection, it should not be used for the diagnosis of low BMD at this time; however, it may be a useful modality in conjunction with traditional projections for the monitoring of $\mathrm{BMD}$ changes over time.

Spine quantitative computerized tomography (QCT) is another method used to measure bone mineral content in AS. It measures volumetric trabecular bone mineral content and excludes cortical bone and other artifacts such as calcified ligaments. Discrepancies between DXA and QCT in AS have been shown particularly in advanced disease, where the majority of patients have normal DXA BMD yet abnormally low QCT [22]. In a small sample size of 15 patients with AS, QCT was shown to decrease over 10 years despite an unchanged BMD by DXA scan [23]. In a multivariate regression analysis adjusting for age and sex, patients with AS had lower BMD at the femoral neck and total hip using high-resolution QCT than patients without AS [4]. Patients with AS have also been shown to have lower cortical volumetric $\mathrm{BMD}$ at the radius and lower trabecular volumetric BMD in the tibia, with trabecular BMD correlating with lumbar spine volumetric BMD [4, 24].

Ultimately, alternate imaging methods for the detection of low BMD have not been validated for clinical use in the detection and monitoring of osteoporosis and osteopenia in the general population nor in AS, though these modalities do surpass some limitations of DXA screening in AS. At this time, we still advocate for the initial use of DXA scan in patients with AS. Especially in patients with early disease in which there is a lower burden of syndesmophytes, DXA is a useful screening modality for the detection of low BMD. We recommend baseline BMD evaluation at the lumbar spine, femoral neck, total hip, and radius.

Elevations in markers of bone turnover have been used to evaluate for increase risk of fracture in patients with low BMD and have been used in some situations to monitor response to osteoporotic therapy [25-28]. However, elevated markers of bone turnover are also seen in active AS and have been associated with the severity of spinal damage $[1,29,30]$. TNF-alpha inhibitor (TNFi) treatment may also modulate levels of bone turnover markers in AS [31]. As bone turnover markers may be affected by disease activity and severity, as well as treatment with TNFi, we do not routinely use bone turnover markers in the evaluation and management of low BMD in AS.

\section{Initial evaluation after detection of low BMD on DXA}

If osteopenia or osteoporosis is identified on initial screening by DXA, we recommend evaluating for secondary causes of low BMD if the patient is under the age of 65 . Specifically, we recommend checking a vitamin D level, thyroid stimulating hormone, parathyroid hormone, basic metabolic panel, celiac serologies, and a hemoglobin A1C for diabetes assessment [32]. If the patient is male, we also recommend obtaining a morning testosterone level to rule out hypogonadism as a contributing cause for low BMD. We assess for estrogen deficiency in women based on clinical history of amenorrhea or early menopause. Proton pump inhibitors, which have been linked with osteoporosis, are commonly prescribed to patients with AS receiving NSAIDS for GI prophylaxis [33]. A change in therapy to H2-antagonists may be considered as an alternative gastric protective agent. Treatment of 
secondary causes of osteoporosis is beyond the scope of this paper and typically warrants referral to an appropriate specialist depending on the condition.

\section{Treatment}

\section{Dietary treatment}

\section{Vitamin D}

Vitamin D is essential for the regulation of calcium homeostasis and for skeletal health. While vitamin D deficiency has been found to occur with greater prevalence in AS in some studies, other studies have failed to show a significant difference from controls [34-36]. In a recent study by Klingberg et al., there was no correlation with 25-OH vitamin D levels and BMD or vertebral fractures in patients with AS [36]. Therefore, at present, there is no guidance in the literature to recommend additional vitamin $\mathrm{D}$ supplementation for the prevention or treatment of low BMD specifically in AS; however, we do follow the Institute of Medicine guidelines regarding the recommended daily intake of calcium and vitamin D and aim for a goal 25-OH vitamin D serum level of at least $40 \mathrm{nmol} /$ $\mathrm{L}$ to minimize fracture risk [37]. We follow the Institute of Medicine guidelines for recommended calcium intake as well [37].

\section{Pharmacologic treatment}

\section{TNF-alpha inhibitors}

TNF-alpha inhibitors (TNFi) are recommended for the treatment of active AS in patients who have failed at least two NSAIDS [38]. The cytokine TNF-alpha has been shown to activate osteoclast-mediated bone resorption; therefore, elevation of TNF-alpha-which is known to occur in AS-may modulate bone loss in AS $[39,40]$. It is postulated that TNFi may act to decrease bone resorption by decreasing TNF-alpha-driven osteoclast stimulation.

In 2008, an open label prospective study of 106 patients with AS showed that treatment with either infliximab or etanercept was associated with a significant increase in BMD at the spine and total hip at 2 years $(p<0.0001$ and $p<0.001$, respectively). Mean BMD change was $5.8 \%$ at the spine and $2.3 \%$ at the hip in comparison to baseline [41]. Similarly, in a prospective observational study of 49 patients with AS by van der Weijden et al., treatment with etanercept was associated with a significant increase in BMD at the spine and hip at 2 years ( $p<0.0001$ and $p=0.014$, respectively) [42]. Despite therapy with etanercept, the number of vertebral fractures more than doubled in 2 years and fracture severity increased. Vertebral fractures were reportedly not associated with BMD or radiographic damage as assessed by mSASSS. No conclusions can be drawn, however, regarding the relative change in fracture risk and severity with etanercept treatment as there were no comparison groups.

In a post hoc analysis of the randomized controlled Ankylosing Spondylitis Study for the Evaluation of Recombinant Infliximab Therapy (ASSERT), a significant increase in $\mathrm{BMD}$ in comparison to baseline was also observed in the spine and hip of patients treated with infliximab for 6 months $(p=0.001$ 
and $p=0.040$, respectively). The median percent increase in spinal BMD scores was $2.5 \%$ in patients treated with infliximab versus $0.5 \%$ in patients treated with placebo $(p=0.001)$, and the median percent increase in hip BMD scores was $0.5 \%$ in patients treated with infliximab versus $0.2 \%$ in patients treated with placebo $(p=0.033)$. These increases in BMD at the spine and hip persisted at week 102 and increases were not related to an increase in syndesmophytes [19]. Incident vertebral fractures were not reported in the post hoc analysis.

The first prospective controlled study comparing changes in BMD in patients with AS treated with TNFi versus AS patients who were not treated with TNFi was published in 2013 [18•]. In this 2-year study, 63 patients were enrolled of which 26 were placed on TNFi (infliximab, etanercept, or adalimumab) at the discretion of the treating rheumatologist. At the end of 2 years, patients who did and did not receive TNFi both had a significant increase in BMD at the spine by DXA in comparison to baseline $(p<0.001$ and $p=0.013$, respectively); the increase in BMD was higher in the group treated with TNFi than the group who did not receive TNFi $(p<0.001$ for spine and $p=0.024$ for total proximal femur). Additionally, patients treated with TNFi had a significant increase in BMD at the proximal femur $(p=0.007)$. Syndesmophyte formation, as measured by the SASSS and the mSASSS, was noted to be greater in the group treated with $\mathrm{TNFi}$, though patients treated with TNFi had higher baseline grade of sacroiliitis suggesting worse disease severity at study onset. Univariate and multivariate analyses revealed an association with increasing SASSS and BMD, which suggests that syndesmophyte formation may account for increases in $\mathrm{BMD}$ at the spine as measured by DXA. However, this would not account for the increase in $\mathrm{BMD}$ at the total proximal femur that was observed in the TNFi-treated group. Similar findings were reported by Li et al. in 2015 in an open label prospective study of 89 patients with AS and low BMD, of whom 42 were treated with TNFi [20]. Patients treated with TNFi had significant increase in BMD at 1 year at the spine $(p<0.0001)$ and femoral neck $(p<0.0001)$; increases at the spine were not secondary to syndesmophytes as evaluated by mSASSS. BMD in the TNFi group was significantly higher than the control group at 1 year $(p<0.0001)$; notably, the control group experienced significant declines in both spine and femoral neck BMD during 1 year $(p<0.0001)$. Two vertebral fractures occurred in the control group, whereas no vertebral fractures occurred in the treatment group at 1 year; due to low numbers, results did not reach significance.

The studies discussed thus far have presented data over the relatively short period of time of 1 to 2 years. In 2013, Durnez et al. published a retrospective study of BMD changes over 6 years in patients with spondyloarthritis treated with TNFi versus a control population of AS patients who did not receive treatment with TNFi [43]. BMD changes were significantly higher in the TNFi group than in the control group at lumbar spine $(p<0.0001)$, femoral neck $(p=0.002)$, and trochanter $(p=$ $0.011)$, but not at total hip $(p=0.062)$ at 6 years; notably, the control group had a decrease in BMD over 6 years while the TNFi-treated group did not. At the lumbar spine, the increase in BMD in TNFi-treated patients was similar regardless of the presence of syndesmophytes. 
However, syndesmophytes were detected using vertebral fracture assessment on DXA and not via validated mSASSS scoring; therefore, results regarding the impact of syndesmophytes on BMD scoring in this study should be interpreted with caution.

There is a paucity of literature regarding the effect of TNFi on the clinical outcomes of reduction in vertebral fractures. Mass et al. recently reported a prospective, observational, cohort study in which 105 patients with AS receiving TNFi were followed for new vertebral fractures over a 4 -year period of time [44•]. Twenty percent of patients developed a new vertebral fracture during the study period. Development of a new vertebral fracture was correlated with older age, smoking, low lumbar spine BMD (Z-score $\leq-2)$, presence of moderate vertebral fractures, and use of anti-osteoporotic treatment at baseline. Those who did develop incident fractures during the study period had significantly less improvement in lumbar spine BMD; no significant changes in BMD between groups were seen at the hip. As this study lacked a control group, no conclusions can be drawn regarding the efficacy of TNFi in reducing incident fractures in AS.

Prospective controlled trials are needed to specifically evaluate the clinical outcome of fracture risk with TNFi treatment in order to assess whether the improvements that are seen in BMD with TNFi treatment translates to improved clinical outcomes in AS patients. While several studies are in agreement that TNFi increase BMD, bone quality may still be affected thus resulting in continued vertebral facture risk. Currently, there is not enough evidence to support the use of TNFi for the sole purpose of preventing loss of BMD in patients with AS. However, we have a low threshold to start TNFi in patients noted to have low BMD and active AS with close monitoring of BMD by DXA at 2-year intervals.

\section{Bisphosphonates}

Bisphosphonates are strong inhibitors of osteoclast activity thus decreasing bone resorption. They are available in both oral and intravenous formulations (Table 1) [45-48]. They are widely used in the treatment of post-menopausal osteoporosis in order to reduce the risk of fractures. The role of bisphosphonates in the treatment of osteoporosis in a younger population and in men is less defined. In a recent network meta-analysis of 36 studies using bisphosphonates for the treatment of primary osteoporosis in both men and women, bisphosphonates significantly reduced the risk of vertebral fracture and non-vertebral fracture with odds ratios and $95 \%$ confidence intervals of $0.54(0.45,0.65)$ and $0.78(0.72,0.84)$, respectively [49]. Specific drugs within the bisphosphonate class were shown to have greater efficacy in prevention of vertebral fractures, with zoledronic acid demonstrating the most effect. Osteoporosis from secondary causes were not included in the analysis thus limiting generalizability to patients with AS. Bisphosphonates have, however, been used in the treatment of inflammatory disease activity in AS, and thus some data regarding their effects on $\mathrm{BMD}$ have been reported in these studies. 


\begin{tabular}{|c|c|c|c|}
\hline Medication & Dose & $\begin{array}{l}\text { Side effects/adverse } \\
\text { reactions }\end{array}$ & Contraindications \\
\hline & \multirow{2}{*}{$\begin{array}{l}\text { Gastrointestinal mucosa } \\
\text { irritation with oral } \\
\text { bisphosphonates }\end{array}$} & \multirow{2}{*}{$\begin{array}{c}\text { Abnormalities of esophagus } \\
\text { (e.g., stricture, achalasia, } \\
\text { esophageal dysmotility) }\end{array}$} \\
\hline Alendronate [34] & $\begin{array}{l}10 \text { mg daily or } 70 \mathrm{mg} \\
\text { weekly }\end{array}$ & & \\
\hline \multirow[t]{2}{*}{ Risedronate [35] } & $5 \mathrm{mg}$ daily or $35 \mathrm{mg}$ & Arthralgias/Myalgias & for oral bisphosphonates \\
\hline & $\begin{array}{l}\text { weekly or } 150 \text { mg } \\
\text { monthly }\end{array}$ & $\begin{array}{l}\text { Hypersensitivity } \\
\text { reaction }\end{array}$ & $\begin{array}{l}\text { Inability to stand or sit } \\
\text { upright after swallowing }\end{array}$ \\
\hline \multirow[t]{2}{*}{ Ibandronate [36] } & \multirow[t]{2}{*}{150 mg monthly } & \multirow{6}{*}{$\begin{array}{l}\text { ONJ } \\
\text { Increased risk of } \\
\text { atypical femoral shaft } \\
\text { fracture with use greater } \\
\text { than 3-5 years }\end{array}$} & for $30-60$ min for oral \\
\hline & & & bisphosphonates \\
\hline \multicolumn{2}{|l|}{ IV Bisphosphonates } & & Hypocalcemia \\
\hline Ibandronate [36] & 3 mg every 3 months & & $\mathrm{CrCl}<35 \mathrm{~mL} / \mathrm{min}$ \\
\hline \multirow[t]{2}{*}{ Zoledronic acid [37] } & 5 mg yearly & & \multirow[t]{2}{*}{ Pregnancy } \\
\hline & & & \\
\hline MAB for RANKL & & Hypocalcemia & Hypocalcemia \\
\hline \multirow[t]{5}{*}{ Denosumab [45] } & \multirow{5}{*}{$\begin{array}{l}60 \text { mg subcutaneous } \\
\text { every } 6 \text { months }\end{array}$} & Hypophosphatemia & \multirow[t]{5}{*}{ Pregnancy } \\
\hline & & Weakness & \\
\hline & & Dyspnea & \\
\hline & & ONJ & \\
\hline & & Infections & \\
\hline
\end{tabular}

In 2014, Viapiani et al. published an open label study of 60 patients with AS who received either IV neridronate (amino-bisphosphonate available in Europe) or infliximab therapy for 6 months. In patients treated with neridronate, a significant increase in BMD was observed at the lumbar spine $(p<0.05$ vs. baseline and vs. infliximab) but not at the hip sites [50•]. Neridronate was as effective as infliximab in reducing disease activity in AS. Of note, the presence or absence of syndesmophytes as a confounding factor was not considered in the analysis.

In a randomized, double-blind, controlled pilot study of 24 patients with AS receiving alendronate versus placebo for 12 months, no difference in BMD was observed between groups [51]. Patients enrolled in this study had normal BMD and a low burden of syndesmophytes upon enrollment. Given the short duration of therapy and small study sample, no specific conclusions can be drawn from this pilot study.

In the 2013 study by Kang et al., a subset of patients also received bisphosphonate therapy. Interestingly, treatment with bisphosphonates in the setting of TNFi increased radiographic progression as measured by SASSS [18 $\bullet$. As only 63 patients with AS were included in this study, of which only 26 patients were treated with $\mathrm{TNFi}$, additional studies are needed to evaluate this paradoxical effect.

While studies are limited in the treatment of low BMD in AS, bisphosphonates have a proven track record in the treatment of osteoporosis with primary outcomes being reduction in fracture risk [49]. Bisphosphonates have been linked with atypical femoral fractures with prolonged use (greater than 3 years for intravenous formulations and 
greater than 5 years for oral formulations) and thus consideration should be made for drug holiday pending clinical assessment of fracture risk at that time [52]. Continued treatment with a bisphosphonate may outweigh the increased risk of atypical femoral fractures in patients with high fracture risk, i.e., patients with a low hip T-score or previous major osteoporotic fracture [52]. Bisphosphonates may not be appropriate for use in women of childbearing age given potential toxic effects on the fetus as evidenced in animal studies [53, 54]. Bisphosphonates have long-term skeletal retention; therefore, their effects may be long-lasting even after discontinuation [55].

\section{Other osteoporosis therapies}

There are two subclasses of osteoporotic agents, which include the antiresorptive agents and the anabolic agents. Bisphosphonates belong to the anti-resorptive class of agents used in the treatment of osteoporosis. Denosumab, a monoclonal antibody against RANKL, is also an antiresorptive agent as it acts to prevent osteoclast formation (Table 1) [56]. RANKL, receptor activator of nuclear factors-kappaB, binds RANK in cell membranes of osteoclasts to stimulate bone resorption. Recently, it was demonstrated that patients with AS have higher levels of serum RANKL. Furthermore, serum RANKL to osteoprotegrin (a decoy receptor for RANKL) ratio was elevated suggesting an imbalance and possible pathogenesis for low BMD in AS [57]. This provides some evidence to support the use of denosumab to treat low BMD in AS; however, there are no studies at present of denosumab in the treatment of low BMD in AS and thus potential benefit is unknown. Given the lack of evidence, we would not recommend denosumab for use as first-line therapy for the treatment of osteoporosis in AS but would consider its use if bisphosphonates are contraindicated.

Teriparatide belongs to a class of anabolic osteoporotic agents and therefore stimulates bone formation. There are no studies evaluating the use of teriparatide in the treatment of osteoporosis in AS. However, as osteoproliferation is a significant complication in AS, agents that promote bone formation may lead to worsening complications, though there are no studies to support this conclusion. Therefore, we do not use teriparatide to treat osteoporosis in patients with AS.

It is widely accepted that weight-bearing exercise improves bone mineral density. There are no studies evaluating the impact of exercise on bone mineral density in patients with ankylosing spondylitis; however, improvements in disease activity and physical function have been noted in patients with AS who participate in exercise programs $[58,59]$. Recent consensus guidelines also encourage consistent physical activity and stretching in patients with AS [60]. It is important that exercise prescription in AS take into account current disease activity and any spinal damage, which may place the patient at greater risk for injury with certain high-impact activities and truncal motions [60]. Clinical practice guidelines vary regarding recommendations on the optimal exercise 


\section{Conclusions}

regimen, frequency, and duration for the treatment of osteoporosis [61]. Most agree that weight-bearing activity is essential, with low-impact activity such as walking meeting this weight-bearing requirement [61]. Muscle strength testing, resistance training, and balance training were also among the most highly recommended physical activities for bone health and prevention of falls in the analyzed clinical practice guidelines [61]. Of note, many studies regarding physical activity for the management of osteoporosis are in post-menopausal women and thus may not fully generalize to the population of patients with AS. However, given the benefits of exercise in patients with AS coupled with benefits of physical activity in osteoporosis treatment/prevention in other demographics, we recommend a general exercise program that emphasizes low weight-bearing activity, core strengthening, balance, and gait training for at least $30 \mathrm{~min}$ on most days of the week.

The burden of low BMD coupled with extensive syndesmophyte formation in AS leads to high vertebral fracture risk. Low BMD and peak fracture risk may occur early after disease diagnosis. Screening for low BMD with standard DXA scan views within 1 year of AS diagnosis is advisable. If low BMD is found, then evaluation for secondary causes of low BMD is recommended. Some patients with longstanding disease may have an overestimation of BMD via traditional AP lumbar DXA screening. Alternate imaging methods to screen for low BMD including lateral lumbar DXA and QCT exist but are not yet validated in AS. We advise managing osteopenia and osteoporosis in patients with AS with nonpharmacological (i.e., general exercise program) and pharmacological treatments (i.e., vitamin $\mathrm{D}$, bisphosphonates). TNFi have been demonstrated to increase $\mathrm{BMD}$ in AS but there is no conclusive evidence supporting that this increase in BMD translates into reduced vertebral fracture risk. The use of TNFi for the sole purpose of managing low BMD in AS is as yet undetermined and warrants further investigation.

\section{Compliance with Ethical Standards}

Conflicts of Interest

AMH declares no conflict of interest. GHL reports scientific advisory board with Janssen.

Human and Animal Rights and Informed Consent

This article does not contain any studies with human or animal subjects performed by any of the authors.

Financial Support and Sponsorship

Research reported in this publication was supported by the National Institute of Arthritis and Musculoskeletal and Skin Diseases of the National Institutes of Health under Award Number T32AR048522. The 
content is solely the responsibility of the authors and does not necessarily represent the official views of the National Institutes of Health.

\section{References and Recommended Reading}

Papers of particular interest, published recently, have been highlighted as:

- Of importance

1. Arends S, Spoorenberg A, Bruyn GA, Houtman PM, Leijsma MK, Kallenberg CGM, et al. The relation between bone mineral density, bone turnover markers, and vitamin D status in ankylosing spondylitis patients with active disease: a cross-sectional analysis. Osteoporos Int. 2011;22:1431-9. Available from: http://www.pubmedcentral.nih.gov/articlerender.fcgi? artid=3073049\&tool=pmcentrez\&rendertype $=$ abstract.

2. El Maghraoui A, Borderie D, Cherruau B, Edouard R, Dougados M, Roux C. Osteoporosis, body composition, and bone turnover in ankylosing spondylitis. J Rheumatol. 1999;26:2205-9.

3. Muntean L, Rojas-Vargas M, Font P, Simon S-P, Rednic $\mathrm{S}$, Schiotis $\mathrm{R}$, et al. Relative value of the lumbar spine and hip bone mineral density and bone turnover markers in men with ankylosing spondylitis. Clin Rheumatol. 2011;30:691-5. Available from: http:// www.ncbi.nlm.nih.gov/pubmed/21221691.

4. Nigil Haroon N, Szabo E, Raboud JM, McdonaldBlumer H, Fung L, Josse RG, et al. Alterations of bone mineral density, bone microarchitecture and strength in patients with ankylosing spondylitis: a cross-sectional study using high-resolution peripheral quantitative computerized tomography and finite element analysis. Arthritis Res Ther. 2015;17:377. Available from: http://arthritis-research.com/content/17/1/377.

5. Van Der Weijden MAC, Claushuis TAM, Nazari T, Lems WF, Dijkmans BAC, Van Der HorstBruinsma IE. High prevalence of low bone mineral density in patients within 10 years of onset of ankylosing spondylitis: A systematic review. Clin Rheumatol. 2012;1529-35.

This review showed that low BMD occurs early in AS, underscoring the importance of early screening.

6. Magrey MN, Lewis S, Asim Khan M. Utility of DXA scanning and Risk Factors for Osteoporosis in Ankylosing Spondylitis; A Prospective Study. Semin Arthritis Rheum. 2016;1-7. Available from: http://linkinghub. elsevier.com/retrieve/pii/S0049017216000937

7. Ghozlani I, Ghazi M, Nouijai A, Mounach A, Rezqi A, Achemlal L, et al. Prevalence and risk factors of osteoporosis and vertebral fractures in patients with ankylosing spondylitis. Bone. 2009;44:772-6. Available from: doi:10.1016/j.bone.2008.12.028.

8. Klingberg E, Geijer M, Göthlin J, Mellström D, Lorentzon M, Hilme E, et al. Vertebral fractures in ankylosing spondylitis are associated with lower bone mineral density in both central and peripheral skeleton. J Rheumatol. 2012;39:12-3.
9. Prieto-Alhambra D, Muñoz-Ortego J, De Vries F, Vosse D, Arden NK, Bowness P, et al. Ankylosing spondylitis confers substantially increased risk of clinical spine fractures: a nationwide case-control study. Osteoporos Int. 2014;26:85-91.

10. Cooper C, Carbone L, Michet CJ, Atkinson EJ, O'Fallon WM, Melton III LJ. Fracture risk in patients with ankylosing spondylitis: a population based study. J Rheumatol. 1994;21:1877-82. Available from: http:// europepmc.org/abstract/MED/7837154.

11. Muñoz-Ortego J, Vestergaard P, Rubio JB, Wordsworth $\mathrm{P}$, Judge A, Javaid MK, et al. Ankylosing spondylitis is associated with an increased risk of vertebral and nonvertebral clinical fractures: a population-based cohort study. J Bone Miner Res. 2014;29:1770-6.

12. Lewiecki EM, Gordon CM, Baim S, Leonard MB, Bishop NJ, Bianchi ML, et al. International Society for Clinical Densitometry 2007 Adult and Pediatric Official Positions. Bone. 2008;43:1115-21. Available from: doi:10.1016/j.bone.2008.08.106.

13. U.S. Preventive Services Task Force. Screening for osteoporosis: U.S. preventive services task force recommendation statement. Ann Intern Med. 2011;154:356-65.

14. Kanis JA, Borgstrom F, De Laet C, Johansson H, Johnell $\mathrm{O}$, Jonsson $\mathrm{B}$, et al. Assessment of fracture risk. Osteoporos Int. 2005;16:581-9. Available from: http:// www.ncbi.nlm.nih.gov/pubmed/15616758.

15. Cosman F, de Beur SJ, LeBoff MS, Lewiecki EM, Tanner B, Randall S, et al. Clinician's guide to prevention and treatment of osteoporosis. Osteoporos Int. 2014;25:2359-81.

16. Watts NB, Adler RA, Bilezikian JP, Drake MT, Eastell R, Orwoll ES, et al. Osteoporosis in men: an Endocrine Society clinical practice guideline. J Clin Endocrinol Metab. 2012;97:1802-22. Available from: http://www. ncbi.nlm.nih.gov/pubmed/22675062.

17. Van Der Weijden MAC, Claushuis TAM, Nazari T, Lems WF, Dijkmans BAC, Van Der Horst-Bruinsma IE. High prevalence of low bone mineral density in patients within 10 years of onset of ankylosing spondylitis: a systematic review. Clin Rheumatol. 2012;31:1529-35.

18. Kang KY, Ju JH, Park S-H, Kim H-Y. The paradoxical effects of TNF inhibitors on bone mineral density and radiographic progression in patients with ankylosing spondylitis. Rheumatology (Oxford). 2013;52:71826. Available from: http://www.ncbi.nlm.nih.gov/ pubmed/23275389.

This study demonstrated increased radiographic severity with TNFi treatment in addition to increased BMD. The TNFi treated 
cohort had more severe sacroiliitis at baseline which may partially explain increased radiographic severity.

19. Visvanathan S, van der Heijde D, Deodhar A, Wagner C, Baker DG, Han J, et al. Effects of infliximab on markers of inflammation and bone turnover and associations with bone mineral density in patients with ankylosing spondylitis. Ann Rheum Dis. 2009;68:17582.

20. Li H, Li Q, Chen X, Ji C, Gu J. Anti-tumor necrosis factor therapy increased spine and femoral neck bone mineral density of patients with active ankylosing spondylitis with low bone mineral density. J Rheumatol. 2015;42:1413-7.

21. Klingberg E, Lorentzon M, Mellström D, Geijer M, Göthlin J, Hilme E, et al. Osteoporosis in ankylosing spondylitis-prevalence, risk factors and methods of assessment. BioMed Central Ltd. 2012;14:R108. Available from: http://www. scopus.com/inward/record.url?eid=2-s2.084860605484 \&partnerID=tZOtx3y1.

22. Lange U, Kluge A, Strunk J, Teichmann J, Bachmann G. Ankylosing spondylitis and bone mineral density - what is the ideal tool for measurement? Rheumatol Int. 2005;26:115-20.

23. Korkosz M, Gąsowski J, Grzanka P, Gorczowski J, Pluskiewicz W, Jeka S, et al. Baseline new bone formation does not predict bone loss in ankylosing spondylitis as assessed by quantitative computed tomography (QCT): 10-year follow-up. BMC Musculoskelet Disord. 2011;12:121. Available from: http:// bmcmusculoskeletdisord.biomedcentral.com/articles/ 10.1186/1471-2474-12-121.

24. Klingberg E, Lorentzon M, Göthlin J, Mellström D, Geijer M, Ohlsson C, et al. Bone microarchitecture in ankylosing spondylitis and the association with bone mineral density, fractures, and syndesmophytes. Arthritis Res Ther. 2013;15:R179. Available from: http:// arthritis-research.com/content/15/6/R179.

25. Vasikaran $S$, Eastell R, Bruyère O, Foldes A, Garnero $\mathrm{P}$, Griesmacher A, et al. Markers of bone turnover for the prediction of fracture risk and monitoring of osteoporosis treatment: a need for international reference standards. Osteoporos Int. 2011;22:391420.

26. Morris HA, Eastell R, Jorgesen NR, Cavalier E, Vasikaran $\mathrm{S}$, Chubb SAP, et al. Clinical usefulness of bone turnover marker concentrations in osteoporosis. Clin Chim Acta. 2016; Available from: http://linkinghub.elsevier. com/retrieve/pii/S0009898116302819

27. Vasikaran S, Cooper C, Eastell R, Griesmacher A, Morris HA, Trenti T, et al. International Osteoporosis Foundation and International Federation of Clinical Chemistry and Laboratory Medicine Position on bone marker standards in osteoporosis. Clin Chem Lab Med. 2011;49:1271-4.

28. Johansson H, Odén A, Kanis JA, McCloskey EV, Morris $\mathrm{HA}$, Cooper C, et al. A meta-analysis of reference markers of bone turnover for prediction of fracture. Calcif. Tissue Int. 2014;94:560-7.
29. Gamez-Nava J, de la Cerda-Trujillo L, Vazquez-Villegas M, Cons-Molina F, Alcaraz-Lopez M, Zavaleta-Muñiz S, et al. Association between bone turnover markers, clinical variables, spinal syndesmophytes and bone mineral density in Mexican patients with ankylosing. Scand J Rheumatol. 2016;9742:1-11. Available from: http://www.tandfonline.com/doi/full/10.3109/ 03009742.2016 .1152399$.

30. Arends S, Spoorenberg A, Efde M, Bos R, Leijsma MK, Bootsma $\mathrm{H}$, et al. Higher bone turnover is related to spinal radiographic damage and low bone mineral density in ankylosing spondylitis patients with active disease: a cross-sectional analysis. PLoS One. 2014;9.

31. Arends S, Spoorenberg A, Houtman PM, Leijsma MK, Bos R, Kallenberg CG, et al. The effect of three years of TNF $\alpha$ blocking therapy on markers of bone turnover and their predictive value for treatment discontinuation in patients with ankylosing spondylitis: a prospective longitudinal observational cohort study. Arthritis Res Ther. 2012;14:R98. Available from: http:// www.pubmedcentral.nih.gov/articlerender.fcgi?artid= $3446472 \&$ tool=pmcentrez\&rendertype $=$ abstract.

32. Mirza F, Canalis E. Management of endocrine disease: secondary osteoporosis: pathophysiology and management. Eur J Endocrinol. 2015;1-52. Available from: http://www.ncbi.nlm.nih.gov/pubmed/25971649

33. Zhou B, Huang Y, Li H, Sun W, Liu J. Proton-pump inhibitors and risk of fractures: an update meta-analysis. Osteoporos Int. 2016;27:339-47.

34. Cai G, Wang L, Fan D, Xin L, Liu L, Hu Y, et al. Vitamin $\mathrm{D}$ in ankylosing spondylitis: review and meta-analysis. Clin Chim Acta. 2015;438:316-22. Available from: http://www.ncbi.nlm.nih.gov/pubmed/25199851.

35. Zhao S, Duffield SJ, Moots RJ, Goodson NJ. Systematic review of association between vitamin $\mathrm{D}$ levels and susceptibility and disease activity of ankylosing spondylitis. Rheumatology. 2014;53:1595-603. Available from: http://www.ncbi.nlm.nih.gov/pubmed/ 24706990.

36. Klingberg E, Oleröd G, Hammarsten O, Forsblad-d'Elia $\mathrm{H}$. The vitamin D status in ankylosing spondylitis in relation to intestinal inflammation, disease activity, and bone health: a cross-sectional study. Osteoporos Int. Osteoporosis International; 2016;1-7. Available from: doi:10.1007/s00198-016-3489-7

37. Ross AC, Taylor CL, Yaktine AL, Del HB. Dietary reference intakes for calcium and vitamin D [Internet]. Instutute Med. 2011. Available from: http://www.nap. edu/catalog/13050/dietary-reference-intakes-forcalcium-and-vitamin-d.

38. Ward MM, Deodhar A, Akl EA, Lui A, Ermann J, Gensler LS, et al. American College of Rheumatology/ Spondylitis Association of America/Spondyloarthritis Research and Treatment Network 2015 Recommendations for the Treatment of Ankylosing Spondylitis and Nonradiographic Axial Spondyloarthritis. Arthritis Rheumatol. 2016;68:282-98.

39. Redlich K, Smolen JS. Inflammatory bone loss: pathogenesis and therapeutic intervention. Nat Rev Drug 
Discov. 2012;11:234-50. Available from: doi:10.1038/ nrd3669.

40. Bal A, Unlu E, Bahar G, Aydog E, Eksioglu E, Yorgancioglu R. Comparison of serum IL-1 beta, sIL2R, IL-6, and TNF-alpha levels with disease activity parameters in ankylosing spondylitis. Clin Rheumatol. 2007;26:211-5. Available from: http://www.ncbi.nlm. nih.gov/pubmed/16583185.

41. Briot K, Gossec L, Kolta S, Dougados M, Roux C. Prospective assessment of body weight, body composition, and bone density changes in patients with spondyloarthropathy receiving anti-tumor necrosis factor-alpha treatment. J Rheumatol. 2008;35:855-61.

42. van der Weijden MAC, van Denderen JC, Lems WF, Nurmohamed MT, Dijkmans BAC, van der HorstBruinsma IE. Etanercept increases bone mineral density in ankylosing spondylitis, but does not prevent vertebral fractures: results of a prospective observational cohort study. J Rheumatol. 2016;43:758-64. Available from: http://www.jrheum.org/cgi/doi/10.3899/ jrheum.150857.

43. Durnez A, Paternotte S, Fechtenbaum J, Landewe RB, Dougados M, Roux C, et al. Increase in bone density in patients with spondyloarthritis during anti-tumor necrosis factor therapy: 6-year followup study. J Rheumatol. 2013;40:1712-8. Available from: http:// www.ncbi.nlm.nih.gov/pubmed/23950191.

44. Maas F, Spoorenberg A, Brouwer E, Schilder AM, Chaudhry RN, Wink F, et al. Radiographic vertebral fractures develop in patients with ankylosing spondylitis during 4 years of TNF-alpha blocking therapy. Clin Exp Rheumatol. 2016;34:191-9.

This study demonstrated that increases in BMD conferred by TNF inhibitors did not translate into prevention of radiographic vertebral fractures.

45. Alendronate Sodium Highlights Of Prescribing Information [Internet]. Whitehouse Station, NJ; Available from: https://www.merck.com/product/usa/pi_ circulars/f/fosamax/fosamax_pi.pdf

46. Risedronate Sodium Highlights Of Prescribing Information [Internet]. Rockaway, NJ; Available from: http://www.allergan.com/assets/pdf/actonel_pi

47. Ibandronate Highlights Of Prescribing Information [Internet]. South San Francisco, CA; Available from: http://www.gene.com/download/pdf/boniva_tablets_ prescribing.pdf

48. Zoledronic Acid Highlights Of Prescribing Information [Internet]. East Hanover, NJ; Available from: https:// www.pharma.us.novartis.com/sites/www.pharma.us. novartis.com/files/reclast.pdf

49. Zhou J, Ma X, Wang T, Zhai S. Comparative efficacy of bisphosphonates in short-term fracture prevention for primary osteoporosis: a systematic review with network meta-analyses. Osteoporos Int. 2016;Available from: http://link.springer.com/10.1007/s00198-0163654-Z

50. Viapiana O, Gatti D, Idolazzi L, Fracassi E, Adami S, Troplini S, et al. Bisphosphonates vs infliximab in ankylosing spondylitis treatment. Rheumatol (United Kingdom). 2014;53:90-4.

This study showed that the amino-bisphosphonate, neridronate, was as effective as infliximab in reducing disease activity in AS and resulted in significantly higher BMD at the lumbar spine in comparison to infliximab after 6 months of therapy.

51. Khabbazi A, Noshad H, Gafarzadeh S, Hajialiloo M, Kolahi S. Alendronate effect on the prevention of bone loss in early stages of ankylosing spondylitis: a randomized, double-blind, placebo-controlled pilot study. Iran Red Crescent Med J. 2014;16:e18022. Available from: http://europepmc.org/articles/ PMC4102986/?report=abstract.

52. Adler R A., Fuleihan GE-H, Bauer DC, Camacho PM, Clarke BL, Clines G a., et al. Managing Osteoporosis in Patients on Long-Term Bisphosphonate Treatment: Report of a Task Force of the American Society for Bone and Mineral Research. J Bone Miner Res. 2015;31:n/a n/a. Available from: http://doi.wiley.com/10.1002/ jbmr.2708

53. Patlas N, Golomb G, Yaffe P, Pinto T, Breuer E, Ornoy A. Transplacental effects of bisphosphonates on fetal skeletal ossification and mineralization in rats. Teratology. 1999;60:68-73.

54. Minsker DH, Manson JM, Peter CP. Effects of the bisphosphonate, alendronate, on parturition in the rat. Toxicol Appl Pharmacol. 1993;121:217-23.

55. Lin JH. Bisphosphonates: A review of their pharmacokinetic properties. Bone. 1996;75-85.

56. Denosumab Highlights Of Prescribing Information [Internet]. Thousand Oaks, CA; Available from: http:// pi.amgen.com/united_states/prolia/prolia_pi.pdf

57. Kim H-R, Lee S-H, Kim H-Y, Kim H-Y. Elevated serum levels of soluble receptor activator of nuclear factorskappaB ligand (sRANKL) and reduced bone mineral density in patients with ankylosing spondylitis (AS). Rheumatology (Oxford). 2006;45:1197-200. Available from: http://www.ncbi.nlm.nih.gov/pubmed/ 16567356.

58. Liang H, Zhang H, Ji H, Wang C. Effects of home-based exercise intervention on health-related quality of life for patients with ankylosing spondylitis: a meta-analysis. Clin Rheumatol. 2015;34:1737-44.

59. O'Dwyer T, O'Shea F, Wilson F. Exercise therapy for spondyloarthritis: a systematic review. Rheumatol Int. 2014;34:887-902.

60. Millner JR, Barron JS, Beinke KM, Butterworth RH, Chasle BE, Dutton LJ, et al. Exercise for ankylosing spondylitis: an evidence-based consensus statement. Semin Arthritis Rheum. 2016;45:411-27. Available from: doi:10.1016/j.semarthrit.2015.08.003.

61. Armstrong JJ, Rodrigues IB, Wasiuta T, MacDermid JC. Quality assessment of osteoporosis clinical practice guidelines for physical activity and safe movement: an AGREE II appraisal. Arch Osteoporos. 2016;11:10-1. 